\title{
Approximately dual frames in Hilbert spaces and applications to Gabor frames
}

\section{Christensen, Ole; Laugesen, Richard S.}

Published in:

Sampling Theory in Signal and Image Processing

Publication date:

2011

Document Version

Early version, also known as pre-print

Link back to DTU Orbit

Citation (APA):

Christensen, O., \& Laugesen, R. S. (2011). Approximately dual frames in Hilbert spaces and applications to Gabor frames. Sampling Theory in Signal and Image Processing, 9, 77-90.

\section{General rights}

Copyright and moral rights for the publications made accessible in the public portal are retained by the authors and/or other copyright owners and it is a condition of accessing publications that users recognise and abide by the legal requirements associated with these rights.

- Users may download and print one copy of any publication from the public portal for the purpose of private study or research.

- You may not further distribute the material or use it for any profit-making activity or commercial gain

- You may freely distribute the URL identifying the publication in the public portal

If you believe that this document breaches copyright please contact us providing details, and we will remove access to the work immediately and investigate your claim. 


\title{
Approximately dual frames in Hilbert spaces and applications to Gabor frames
}

\author{
Ole Christensen and Richard S. Laugesen
}

October 22, 2010

\begin{abstract}
Approximately dual frames are studied in the Hilbert space setting. Approximate duals are easier to construct than classical dual frames, and can be tailored to yield almost perfect reconstruction.

Bounds on the deviation from perfect reconstruction are obtained for approximately dual frames constructed via perturbation theory. An alternative bound is derived for the rich class of Gabor frames, by using the Walnut representation of the frame operator to estimate the deviation from equality in the duality conditions.

To illustrate these results, we construct explicit approximate duals of Gabor frames generated by the Gaussian; these approximate duals yield almost perfect reconstruction. Surprisingly, the method applies also to certain Gabor frames that are far from being tight.
\end{abstract}

\section{Introduction}

Let $\mathcal{H}$ be a separable Hilbert space with inner product $\langle\cdot, \cdot\rangle$. Given a frame $\left\{f_{k}\right\}$ for $\mathcal{H}$, it is well known that there exists at least one dual frame $\left\{g_{k}\right\}$, that is, a frame for which

$$
f=\sum\left\langle f, f_{k}\right\rangle g_{k}, \quad \forall f \in \mathcal{H}
$$

Unfortunately, it is usually complicated to calculate a dual frame explicitly. Hence we seek methods for constructing approximate duals.

AMS MSC: 42C15. Key Words: Frames, approximate duals, Gabor frames, Gaussian. 
An approximately dual frame $\left\{g_{k}\right\}$ associated with $\left\{f_{k}\right\}$ satisfies

$$
\left\|f-\sum\left\langle f, f_{k}\right\rangle g_{k}\right\| \leq \epsilon\|f\|, \quad \forall f \in \mathcal{H}
$$

for some $\epsilon<1$; here $\epsilon$ measures the deviation from equality in the frame duality condition. The bound (1) is particularly interesting when $\epsilon$ is small, but for any $\epsilon<1$ we will obtain a family of frames that interpolate between the approximately dual frame $\left\{g_{k}\right\}$ and a classical dual frame.

We further use perturbation ideas to construct approximately dual frames. There are situations where it is hard to find a dual frame for a given frame $\left\{f_{k}\right\}$, but where $\left\{f_{k}\right\}$ lies close to a frame $\left\{h_{k}\right\}$ for which a dual frame $\left\{g_{k}\right\}$ is known explicitly. We present conditions under which such a frame $\left\{g_{k}\right\}$ is approximately dual to $\left\{f_{k}\right\}$, both in the case where $\left\{g_{k}\right\}$ is an arbitrary dual of $\left\{h_{k}\right\}$ and for the particular case where it is the canonical dual.

Such general Hilbert space estimates might of course be improved for concrete classes of frames. For Gabor frames in $L^{2}(\mathbb{R})$ we present a better, direct argument for obtaining an inequality of type (1), based on the Walnut representation of the frame operator. By this method, we construct approximately dual frames for two Gabor frames generated by the Gaussian, obtaining very small values for $\epsilon$.

Note that the idea of approximately dual frames has appeared previously, especially for wavelets [2], [7], [9, Section 2.13], Gabor systems [1], [6, Sections $3,4]$, [16], in the general context of coorbit theory [5], and in sensor modeling [13]. The paper [13] also deals with frames in general Hilbert spaces, and we will comment on the connections with the present paper in Section 4.

\section{Basic frame theory}

A sequence $\left\{f_{k}\right\}$ in $\mathcal{H}$, indexed by an arbitrary countable index set, is a frame if there exist constants $A, B>0$ such that

$$
A\|f\|^{2} \leq \sum\left|\left\langle f, f_{k}\right\rangle\right|^{2} \leq B\|f\|^{2} \quad \forall f \in \mathcal{H} .
$$

Any numbers $A, B$ such that (2) holds are called (lower and upper) frame bounds. For the introductory frame material that follows, see any standard reference on frames, such as [4], [17], [3, Chapter 5].

If the upper inequality in (2) holds, then $\left\{f_{k}\right\}$ is a Bessel sequence. In that case so is $\left\{W f_{k}\right\}$, for any bounded operator $W: \mathcal{H} \rightarrow \mathcal{H}$. 
Given a Bessel sequence $\left\{f_{k}\right\}$, the synthesis operator $T: \ell^{2} \rightarrow \mathcal{H}$ given by

$$
T\left\{c_{k}\right\}=\sum c_{k} f_{k}
$$

is linear and bounded, with $\|T\| \leq \sqrt{B}$; the series converges unconditionally for all $\left\{c_{k}\right\} \in \ell^{2}$. The adjoint of $T$ is the analysis operator $T^{*}: \mathcal{H} \rightarrow \ell^{2}$ given by

$$
T^{*} f=\left\{\left\langle f, f_{k}\right\rangle\right\}
$$

Given a Bessel sequence $\left\{f_{k}\right\}$, a Bessel sequence $\left\{g_{k}\right\}$ is called a dual frame if

$$
f=\sum\left\langle f, f_{k}\right\rangle g_{k}, \quad \forall f \in \mathcal{H} .
$$

Condition (3) means that analysis using $\left\{f_{k}\right\}$ followed by synthesis using $\left\{g_{k}\right\}$ yields the identity operator. We then adopt the terminology of signal processing and speak about perfect reconstruction.

The frame operator is $T T^{*}$, which is well defined and bounded whenever $\left\{f_{k}\right\}$ is a Bessel sequence. If $\left\{f_{k}\right\}$ is a frame, then one choice of dual is the canonical dual frame $\left\{\left(T T^{*}\right)^{-1} f_{k}\right\}$. When $\left\{f_{k}\right\}$ is redundant, infinitely many other dual frames exist.

\section{Approximate duality}

In order to apply the dual frame expansion (3), we first need a dual frame. Unfortunately, it might be cumbersome - or even impossible - to calculate a dual frame explicitly. In the literature one finds only a few infinite dimensional non-tight frames for which a dual has been constructed. This paucity of constructions leads us to seek frames that are "close to dual".

Suppose that $\left\{f_{k}\right\}$ and $\left\{g_{k}\right\}$ are Bessel sequences in $\mathcal{H}$. Denote their synthesis operators by $T: \ell^{2} \rightarrow \mathcal{H}$ and $U: \ell^{2} \rightarrow \mathcal{H}$, respectively. Then

$$
T U^{*} f=\sum\left\langle f, g_{k}\right\rangle f_{k}, \quad f \in \mathcal{H},
$$

and

$$
U T^{*} f=\sum\left\langle f, f_{k}\right\rangle g_{k}, \quad f \in \mathcal{H} .
$$

These operators are called mixed frame operators. In terms of these operators, the Bessel sequences $\left\{f_{k}\right\}$ and $\left\{g_{k}\right\}$ are dual frames if and only if $T U^{*}=I$ or $U T^{*}=I$. 
Definition 3.1 Two Bessel sequences $\left\{f_{k}\right\}$ and $\left\{g_{k}\right\}$ are approximately dual frames if $\left\|I-T U^{*}\right\|<1$ or $\left\|I-U T^{*}\right\|<1$.

This notion of approximately dual frames does not incorporate the natural desire for (1) to hold with a small value of $\epsilon$ : it is only guaranteed that (1) holds with some $\epsilon<1$. However, as we will see in Proposition 3.2, there is an inductive procedure that leads us arbitrarily close to perfect reconstruction, based on any pair of approximately dual frames.

The two conditions in Definition 3.1 are equivalent, by taking adjoints. Note that if $\left\{f_{k}\right\}$ and $\left\{g_{k}\right\}$ are approximately dual frames, then the operator $U T^{*}$ is invertible, and each $f \in \mathcal{H}$ has the representation

$$
f=\left(U T^{*}\right)^{-1} U T^{*} f=\sum\left\langle f, f_{k}\right\rangle\left(U T^{*}\right)^{-1} g_{k} .
$$

From here, a standard argument shows that $\left\{f_{k}\right\}$ is a frame, with dual $\left\{\left(U T^{*}\right)^{-1} g_{k}\right\}$. By symmetry, $\left\{g_{k}\right\}$ is a frame as well.

Proposition 3.2 Assume that $\left\{f_{k}\right\}$ and $\left\{g_{k}\right\}$ are approximately dual frames. Then the following hold:

(i) The dual frame $\left\{\left(U T^{*}\right)^{-1} g_{k}\right\}$ of $\left\{f_{k}\right\}$ can be written

$$
\left(U T^{*}\right)^{-1} g_{k}=g_{k}+\sum_{n=1}^{\infty}\left(I-U T^{*}\right)^{n} g_{k}
$$

(ii) For fixed $N \in \mathbb{N}$, consider the corresponding partial sum,

$$
\gamma_{k}^{(N)}=g_{k}+\sum_{n=1}^{N}\left(I-U T^{*}\right)^{n} g_{k}
$$

Then $\left\{\gamma_{k}^{(N)}\right\}$ is an approximate dual of $\left\{f_{k}\right\}$. Denoting its associated synthesis operator by $Z_{N}$, we have

$$
\left\|I-Z_{N} T^{*}\right\| \leq\left\|I-U T^{*}\right\|^{N+1} \rightarrow 0 \quad \text { as } N \rightarrow \infty .
$$

Proof. Assuming that $\left\{f_{k}\right\}$ and $\left\{g_{k}\right\}$ are approximately dual frames, the inverse of $U T^{*}$ can be written via a Neumann series as

$$
\left(U T^{*}\right)^{-1}=\left(I-\left(I-U T^{*}\right)\right)^{-1}=\sum_{n=0}^{\infty}\left(I-U T^{*}\right)^{n} .
$$


The result in (i) now follows. For (ii), note that $\left\{\gamma_{k}^{(N)}\right\}$ is a Bessel sequence since it is obtained from the Bessel sequence $\left\{g_{k}\right\}$ by a bounded transformation. And

$$
\begin{aligned}
Z_{N} T^{*} f=\sum\left\langle f, f_{k}\right\rangle \gamma_{k}^{(N)} & =\sum\left\langle f, f_{k}\right\rangle \sum_{n=0}^{N}\left(I-U T^{*}\right)^{n} g_{k} \\
& =\sum_{n=0}^{N}\left(I-U T^{*}\right)^{n} U T^{*} f \\
& =\sum_{n=0}^{N}\left(I-U T^{*}\right)^{n}\left(I-\left(I-U T^{*}\right)\right) f \\
& =f-\left(I-U T^{*}\right)^{N+1} f
\end{aligned}
$$

by telescoping. Thus

$$
\begin{aligned}
\left\|I-Z_{N} T^{*}\right\| & =\left\|\left(I-U T^{*}\right)^{N+1}\right\| \\
& \leq\left\|I-U T^{*}\right\|^{N+1}<1 .
\end{aligned}
$$

The approximately dual frame $\left\{g_{k}\right\}$ can be regarded as a zero-th order approximation to the (exact) dual frame $\left\{\left(U T^{*}\right)^{-1} g_{k}\right\}$, in part (i) of Proposition 3.2. In case $\left\|I-U T^{*}\right\|$ is small, reconstruction using the approximate dual $\left\{g_{k}\right\}$ gives close to perfect reconstruction. Part (ii) of the proposition yields a family of approximately dual frames that interpolates between the approximate dual $\left\{g_{k}\right\}$ and the dual frame $\left\{\left(U T^{*}\right)^{-1} g_{k}\right\}$; by choosing $N$ sufficiently large, we can obtain a reconstruction that is "almost perfect". The drawback of the result with respect to potential applications is the complicated structure of the operator in (4) defining the sequence $\gamma_{k}^{(N)}$.

\section{Perturbation and approximately dual frames}

Consider approximately dual frames $\left\{f_{k}\right\}$ and $\left\{g_{k}\right\}$, with associated synthesis operators $T$ and $U$, respectively. To motivate the results, we note once more that it can be a nontrivial task to find the canonical dual frame (or any other dual) associated with $\left\{f_{k}\right\}$. We seek to connect this fact with perturbation theory by asking the following question: if we can find a frame $\left\{h_{k}\right\}$ that 
is close to $\left\{f_{k}\right\}$ and for which it is possible to find a dual frame $\left\{g_{k}\right\}$, does it follow that $\left\{g_{k}\right\}$ is an approximate dual of $\left\{f_{k}\right\}$ ? We will present some sufficient conditions for an affirmative answer.

First we state a general result valid for dual frames $\left\{g_{k}\right\}$ with sufficiently small Bessel bound (Proposition 4.1); later we state a more explicit consequence for the case where $\left\{g_{k}\right\}$ is the canonical dual frame (Proposition 4.3).

Technically, we need not assume that $\left\{f_{k}\right\}$ is a frame in the next result, because the frame property follows as a conclusion. On the other hand, as explained above, the main use of the result is when $\left\{f_{k}\right\}$ is known in advance to be a frame.

Proposition 4.1 (Dual of perturbed sequence) Assume that $\left\{f_{k}\right\}$ is a sequence in $\mathcal{H}$ and that $\left\{h_{k}\right\}$ is a frame for which

$$
\sum\left|\left\langle f, f_{k}-h_{k}\right\rangle\right|^{2} \leq R\|f\|^{2}, \quad \forall f \in \mathcal{H},
$$

for some $R>0$. Consider a dual frame $\left\{g_{k}\right\}$ of $\left\{h_{k}\right\}$ with synthesis operator $U$, and assume $\left\{g_{k}\right\}$ has upper frame bound $C$.

If $C R<1$ then $\left\{f_{k}\right\}$ and $\left\{g_{k}\right\}$ are approximately dual frames, with

$$
\left\|I-U T^{*}\right\| \leq\|U\| \sqrt{R} \leq \sqrt{C R}<1 .
$$

Proof. With our usual notation, we have $U V^{*}=I$ since $\left\{g_{k}\right\}$ and $\left\{h_{k}\right\}$ are dual frames. Hence

$$
\left\|I-U T^{*}\right\|=\left\|U\left(V^{*}-T^{*}\right)\right\| \leq\|U\|\left\|V^{*}-T^{*}\right\| \leq \sqrt{C R}<1 .
$$

It is crucial in Proposition 4.1 that the dual frame $\left\{g_{k}\right\}$ has upper frame bound less than $1 / R$. Otherwise $\left\{g_{k}\right\}$ need not be an approximate dual of $\left\{f_{k}\right\}$, as the next example shows.

Example 4.2 Consider $\mathcal{H}=\mathbb{C}^{2}$ with the standard basis $\left\{e_{1}, e_{2}\right\}$. Let $\epsilon>0$ and consider the frames

$$
\left\{f_{k}\right\}=\left\{0, e_{1}, e_{2}\right\}, \quad\left\{h_{k}\right\}=\left\{\epsilon e_{1}, e_{1}, e_{2}\right\}, \quad\left\{g_{k}\right\}=\left\{\epsilon^{-1} e_{1}, 0, e_{2}\right\} .
$$

Write $T, V, U$ for the associated synthesis operators. Note that

$$
T U^{*} f=\left\langle f, e_{2}\right\rangle e_{2} ;
$$


this operator is neither injective nor surjective, and so $\left\{f_{k}\right\}$ and $\left\{g_{k}\right\}$ are not approximate dual frames. Clearly, $\left\{h_{k}\right\}$ is a frame for $\mathbb{C}^{2}$, regardless of the choice of $\epsilon$. Now, because

$$
\sum_{k=1}^{3}\left|\left\langle f, f_{k}-h_{k}\right\rangle\right|^{2}=\left|\left\langle f, \epsilon e_{1}\right\rangle\right|^{2} \leq \epsilon^{2}\|f\|^{2}, \quad \forall f \in \mathbb{C}^{2},
$$

the condition in Proposition 4.1 is satisfied with $R=\epsilon^{2}$. But no matter how close $\left\{h_{k}\right\}$ gets to $\left\{f_{k}\right\}$ (meaning, no matter how small $\epsilon$ is), the frame $\left\{g_{k}\right\}$ is not an approximate dual of $\left\{f_{k}\right\}$. Note that for $\epsilon<1$, the frame $\left\{g_{k}\right\}$ has the upper frame bound $C=\epsilon^{-2}=1 / R$; thus, Proposition 4.1 is not contradicted.

For practical applications of Proposition 4.1, it is problematic that the possible values of $R$ in (6) are related to the upper bound of $\left\{g_{k}\right\}$. By letting $\left\{g_{k}\right\}$ be the canonical dual of $\left\{h_{k}\right\}$ this problem is avoided:

Proposition 4.3 (Canonical dual of perturbed frame) Let $\left\{f_{k}\right\}$ be a frame for $\mathcal{H}$ with frame bounds $A, B$. Let $\left\{h_{k}\right\}$ be a sequence in $\mathcal{H}$ for which

$$
\sum\left|\left\langle f, f_{k}-h_{k}\right\rangle\right|^{2} \leq R\|f\|^{2}, \quad \forall f \in \mathcal{H},
$$

for some $R<A / 4$. Denote the synthesis operator for $\left\{h_{k}\right\}$ by $V$.

Then $\left\{h_{k}\right\}$ is a frame. Its canonical dual frame $\left\{g_{k}\right\}=\left\{\left(V V^{*}\right)^{-1} h_{k}\right\}$ is an approximate dual of $\left\{f_{k}\right\}$ with

$$
\left\|I-U T^{*}\right\| \leq \frac{1}{\sqrt{A / R}-1}<1
$$

where $U$ denotes the synthesis operator for $\left\{g_{k}\right\}$.

Proof. The sequence $\left\{h_{k}\right\}$ is a frame with frame bounds $(\sqrt{A}-\sqrt{R})^{2}$ and $(\sqrt{B}+\sqrt{R})^{2}$, by [3, Corollary 5.6.3]. The canonical dual frame of $\left\{h_{k}\right\}$ is $\left\{g_{k}\right\}=\left\{\left(V V^{*}\right)^{-1} h_{k}\right\}$, with frame bounds

$$
\frac{1}{(\sqrt{B}+\sqrt{R})^{2}} \text { and } \frac{1}{(\sqrt{A}-\sqrt{R})^{2}}
$$

by [3, Lemma 5.1.6]. In terms of the synthesis operator $U$ for $\left\{g_{k}\right\}$, the upper bound says

$$
\|U\| \leq \frac{1}{\sqrt{A}-\sqrt{R}}
$$


Proposition 4.1 therefore implies

$$
\begin{aligned}
\left\|I-U T^{*}\right\| & \leq\|U\| \sqrt{R} \\
& \leq \frac{\sqrt{R}}{\sqrt{A}-\sqrt{R}} \\
& =\frac{1}{\sqrt{A / R}-1} .
\end{aligned}
$$

To complete the proof, just notice this last expression is smaller than 1 if and only if $R<A / 4$.

We note that the idea of approximate duals also appear in the paper [13] by Li and Yan. The motivation in [13] comes from sensor modeling, but they treat approximate duals from the mathematical point of view. Technically, the approach in [13] is different from the one presented here. In particular, [13] does not apply a condition of the form (6), which implies that it is necessary to assume $\left\{f_{k}\right\}$ as well as $\left\{h_{k}\right\}$ to be a frame. In [13], it is just assumed that $\left\|f_{k}-h_{k}\right\|$ is sufficiently small for all $k$; this does not imply that $\left\{h_{k}\right\}$ is a frame whenever $\left\{f_{k}\right\}$ is a frame. In addition, [13] applies a decay condition on the inner products $\left\langle f_{k}-h_{k}, f_{\ell}-h_{\ell}\right\rangle$ which does not appear in our work.

\section{Gabor frames and approximate duals}

A Gabor frame is a frame for $L^{2}(\mathbb{R})$ of the form

$$
\left\{e^{2 \pi i m b} g(x-n a)\right\}_{m, n \in \mathbb{Z}}
$$

for suitably chosen parameters $a, b>0$ and a fixed function $g \in L^{2}(\mathbb{R})$, called the window function. The number $a$ is called the translation parameter and $b$ is the modulation parameter. Introducing the operators

$$
\left(T_{a} g\right)(x)=g(x-a),\left(E_{b} g\right)(x)=e^{2 \pi i b x} g(x), \quad \text { for } a, b, x \in \mathbb{R},
$$

the Gabor system can be written in the short form $\left\{E_{m b} T_{n a} g\right\}_{m, n \in \mathbb{Z}}$. For more information on Gabor analysis and its role in time-frequency analysis we refer to the book by Gröchenig [8].

Recall the duality conditions for a pair of Gabor systems, due to Janssen [10] and Ron and Shen [14]: 
Lemma 5.1 Two Bessel sequences $\left\{E_{m b} T_{n} \varphi\right\}_{m, n \in \mathbb{Z}}$ and $\left\{E_{m b} T_{n} g\right\}_{m, n \in \mathbb{Z}}$ form dual frames for $L^{2}(\mathbb{R})$ if and only if the equations

$$
\begin{aligned}
\sum_{k \in \mathbb{Z}} \overline{\varphi(x-a k)} g(x-a k)-b & =0, \\
\sum_{k \in \mathbb{Z}} \overline{\varphi(x-n / b-a k)} g(x-a k) & =0, \quad n \in \mathbb{Z} \backslash\{0\},
\end{aligned}
$$

hold a.e.

Recall that the Wiener space $W$ consists of all bounded measurable functions $g: \mathbb{R} \rightarrow \mathbb{C}$ for which

$$
\sum_{n \in \mathbb{Z}}\left\|g \chi_{[n, n+1[}\right\|_{\infty}<\infty
$$

It is well known that if $g \in W$ then $\left\{E_{m b} T_{n a} g\right\}_{m, n \in \mathbb{Z}}$ is a Bessel sequence for each choice of $a, b>0$.

Proposition 5.2 Given two functions $\varphi, g \in W$ and two parameters $a, b>$ 0 , let $T$ denote the synthesis operator associated with the Gabor system $\left\{E_{m b} T_{n a} \varphi\right\}_{m, n \in \mathbb{Z}}$, and $U$ the synthesis operator associated with $\left\{E_{m b} T_{n a} g\right\}_{m, n \in \mathbb{Z}}$. Then

$$
\left\|I-U T^{*}\right\| \leq \frac{1}{b}\left[\left\|b-\sum_{k \in \mathbb{Z}} \overline{T_{a k} g} T_{a k} \varphi\right\|_{\infty}+\sum_{n \neq 0}\left\|\sum_{k \in \mathbb{Z}} \overline{T_{n / b} T_{a k} g} T_{a k} \varphi\right\|_{\infty}\right] .
$$

Proof. The starting point is the Walnut representation [15] of the mixed frame operator associated with $\left\{E_{m b} T_{n a} \varphi\right\}_{m, n \in \mathbb{Z}}$ and $\left\{E_{m b} T_{n a} g\right\}_{m, n \in \mathbb{Z}}$. According to Theorem 6.3.2 in [8],

$$
U T^{*} f(\cdot)=\frac{1}{b} \sum_{n \in \mathbb{Z}}\left(\sum_{k \in \mathbb{Z}} \overline{T_{a k} \varphi(\cdot-n / b)} T_{a k} g(\cdot)\right) T_{n / b} f(\cdot), \quad f \in L^{2}(\mathbb{R}) .
$$


Thus,

$$
\begin{aligned}
& \left\|f-U T^{*} f\right\| \\
\leq & \left\|\left(1-\frac{1}{b} \sum_{k \in \mathbb{Z}} \overline{T_{a k} \varphi(\cdot)} T_{a k} g(\cdot)\right) f\right\| \\
& +\frac{1}{b}\left\|\sum_{n \neq 0}\left(\sum_{k \in \mathbb{Z}} \overline{T_{a k} \varphi(\cdot-n / b)} T_{a k} g(\cdot)\right) T_{n / b} f\right\| \\
\leq & \frac{1}{b}\left\|b-\sum_{k \in \mathbb{Z}} \overline{T_{a k} \varphi} T_{a k} g\right\|_{\infty}\|f\|+\frac{1}{b} \sum_{n \neq 0}\left\|\sum_{k \in \mathbb{Z}} \overline{T_{n / b} T_{a k} \varphi} T_{a k} g\right\|_{\infty}\|f\|,
\end{aligned}
$$

which concludes the proof.

Observe that the terms appearing in the estimate in Proposition 5.2 measure the deviation from equality in the duality relations in Lemma 5.1, with respect to the $\|\cdot\|_{\infty}$-norm. In particular, in case of perfect reconstruction our estimate gives the optimal result $\left\|I-U T^{*}\right\|=0$.

\section{Applications to Gabor frames generated by the Gaussian}

The Gaussian is well known to generate a Gabor frame whenever $a b<1$. With the scaling chosen as

$$
\varphi(x):=2^{1 / 4} e^{-\pi x^{2}},
$$

it was shown by Janssen [11] that for any $\epsilon>0, \epsilon<1-a b$, the function

$\gamma_{\epsilon}(x):=2^{-1 / 4} b K^{-1} e^{\pi x^{2}} \sum_{k \in \mathbb{Z}}(-1)^{k} e^{-\pi a(k+1 / 2)^{2} / b} \operatorname{erfc}[(x-(k+1 / 2) a) \sqrt{\pi / \epsilon}]$

with

$$
K:=\sum_{k \in \mathbb{Z}}(-1)^{k}(2 k+1) e^{-\pi a(k+1 / 2)^{2}}, \quad \operatorname{erfc}(x):=\frac{2}{\sqrt{\pi}} \int_{x}^{\infty} e^{-s^{2}} d s
$$

generates a dual frame of $\left\{E_{m b} T_{n a} \varphi\right\}_{m, n \in \mathbb{Z}}$.

The expression (9) is appealing, but quite complicated, and so it is natural to ask for approximately dual frames with a simpler expression. 
We will construct explicit, approximately dual frames associated with the Gaussian, for certain choices of $a$ and $b$. These approximately dual frames provide almost perfect reconstruction; Example 6.1 is particularly interesting because it deals with a frame that is far from being tight, i.e., no easy way of obtaining an approximately dual frame is available.

\section{Example 6.1 Let}

$$
\varphi(x)=e^{-4 x^{2}} .
$$

An application of Theorem 5.1.5 in [3] shows that the functions $\left\{E_{0.1 m} T_{n} \varphi\right\}_{m, n \in \mathbb{Z}}$ form a frame for $L^{2}(\mathbb{R})$ with frame bounds $A=2.6, B=10.1$. Let $h=B_{8}$ denote the eighth-order B-spline, centered at the origin. The function

$$
h(x)=\frac{315}{151} B_{8}(2.36 x)
$$

yields a close approximation of $\varphi$, see Figure 1(a). A numerical calculation based on Theorem 5.1.5 in [3] shows that the functions $\left\{E_{0.1 m} T_{n}(\varphi-h)\right\}_{m, n \in \mathbb{Z}}$ form a Bessel sequence with Bessel bound $R=6.5 \cdot 10^{-4}<A / 4$.

The function $h$ has support on $[-4 / 2.36,4 / 2.36]$, an interval of length $8 / 2.36$. Since the modulation parameter $b=0.1$ is smaller than $(8 / 2.36)^{-1}$ and the function

$$
H(x)=\sum_{k \in \mathbb{Z}}|h(x+k)|^{2}
$$

is bounded above and below away from zero, it follows from Corollary 9.1.7 in [3] that the frame $\left\{E_{0.1 m} T_{n} h\right\}_{m, n \in \mathbb{Z}}$ has the canonical dual frame $\left\{E_{0.1 m} T_{n} g\right\}_{m, n \in \mathbb{Z}}$, where

$$
\begin{aligned}
g(x) & =\frac{0.1}{\sum_{n \in \mathbb{Z}}|h(x+n)|^{2}} h(x) \\
& =\frac{15.1}{315} \frac{1}{\sum_{n \in \mathbb{Z}}\left|B_{8}(2.36(x+n))\right|^{2}} B_{8}(2.36 x) .
\end{aligned}
$$

See Figure 1(b).

The frame $\left\{E_{0.1 m} T_{n} g\right\}_{m, n \in \mathbb{Z}}$ is approximately dual to $\left\{E_{0.1 m} T_{n} \varphi\right\}_{m, n \in \mathbb{Z}}$, by Proposition 4.3. Denoting their synthesis operators by $U$ and $T$ respectively, the approximation rate in Proposition 4.3 is measured by

$$
\left\|I-U T^{*}\right\| \leq \frac{1}{\sqrt{A / R}-1} \leq 0.016 .
$$




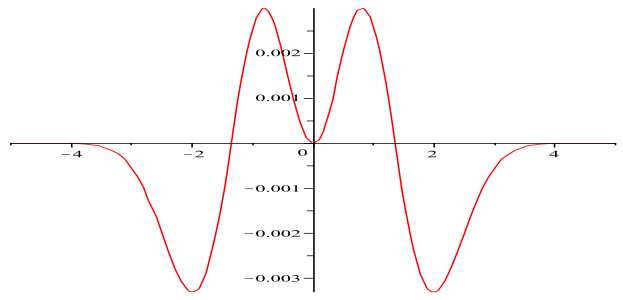

(a)

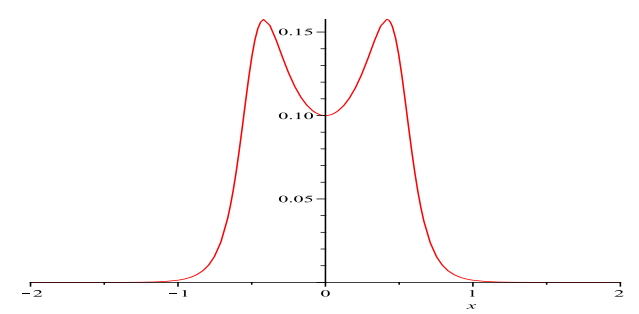

(b)

Figure 1: (a): The function $x \mapsto e^{-4 x^{2}}-\frac{315}{151} B_{8}(2.36 x)$; (b): the approximately dual window $g$ in $(10)$.

On the other hand, Proposition 5.2 yields the somewhat better estimate

$$
\left\|I-U T^{*}\right\| \leq \frac{1}{\sqrt{A / R}-1} \leq 0.009 .
$$

Thus the approximate dual frame almost yields perfect reconstruction.

Note that the frame $\left\{E_{0.1 m} T_{n} \varphi\right\}_{m, n \in \mathbb{Z}}$ is far from being tight. Thus, the sequence $\left\{E_{0.1 m} T_{n}\left(\frac{2}{A+B} \varphi\right)\right\}_{m, n \in \mathbb{Z}}$ is a poor approximate dual: the standard estimate

$$
\left\|I-\frac{2}{A+b} S\right\| \leq \frac{B-A}{B+A}
$$

yields

$$
\left\|I-\frac{2}{A+B} S\right\| \leq \frac{\frac{B}{A}-1}{\frac{B}{A}+1}=0.59,
$$

which is far worse than the result in (12).

Remark 6.2 In case a closer approximation than in (12) is required, an application of the iterative procedure in Proposition 3.2 (ii) can bring us as close to perfect reconstruction as desired. Letting $\left\{f_{k}\right\}$ be a Gabor frame $\left\{E_{m b} T_{n a} f\right\}_{m, n \in \mathbb{Z}}$ and $\left\{g_{k}\right\}$ be an approximately dual Gabor frame $\left\{E_{m b} T_{n a} g\right\}_{m, n \in \mathbb{Z}}$, each approximate dual $\left\{\gamma_{k}^{(N)}\right\}$ constructed in Proposition 3.2 will again have Gabor structure. For $N=1$, an easy calculation shows 
that $\left\{\gamma_{k}^{(N)}\right\}$ will be the Gabor system $\left\{E_{m b} T_{n a} \gamma\right\}_{m, n \in \mathbb{Z}}$, where

$$
\begin{aligned}
\gamma=g+\left(I-U T^{*}\right) g & =2 g-U T^{*} g \\
& =2 g-\sum_{m^{\prime}, n^{\prime} \in \mathbb{Z}}\left\langle g, E_{m^{\prime} b} T_{n^{\prime} a} f\right\rangle E_{m^{\prime} b} T_{n^{\prime} a} g \\
& =(2-\langle g, f\rangle) g-\sum_{\left(m^{\prime}, n^{\prime}\right) \neq(0,0)}\left\langle g, E_{m^{\prime} b} T_{n^{\prime} a} f\right\rangle E_{m^{\prime} b} T_{n^{\prime} a} g .
\end{aligned}
$$

For the particular case considered in Example 6.1, and denoting the synthesis operator for $\left\{E_{m b} T_{n a} \gamma\right\}_{m, n \in \mathbb{Z}}$ by $Z$, Proposition 3.2 shows that the estimate in (11) will be replaced by

$$
\left\|I-Z^{*} T\right\| \leq 0.009^{2}=8.1 \times 10^{-5} .
$$

\section{Acknowledgments}

Laugesen thanks the Department of Mathematics at the Technical University of Denmark for travel support and a warm welcome. Both authors thank the reviewers for their helpful comments and for informing us about related literature.

\section{References}

[1] P. Balazs, H. G. Feichtinger, M. Hampejs and G. Kracher: Double preconditioning for Gabor frames. IEEE Trans. Signal Process. 54, 45974610, 2006.

[2] H.-Q. Bui and R. S. Laugesen: Frequency-scale frames and the solution of the Mexican hat problem. Constr. Approx., to appear.

[3] O. Christensen: Frames and bases. An introductory course. Birkhäuser, Boston, 2008.

[4] I. Daubechies: Ten lectures on wavelets. SIAM, Philadelphia, 1992.

[5] H. G. Feichtinger and K. Gröchenig: Banach spaces related to integrable group representations and their atomic decomposition I. J. Funct. Anal. 86 (1989), 307-340. 
[6] H. G. Feichtinger and N. Kaiblinger. Varying the time-frequency lattice of Gabor frames. Trans. Amer. Math. Soc. 356, 2001-2023, 2004.

[7] J. E. Gilbert, Y. S. Han, J. A. Hogan, J. D. Lakey, D. Weiland and G. Weiss. Smooth molecular decompositions of functions and singular integral operators. Mem. Amer. Math. Soc. 156, no. 742, 74 pp., 2002.

[8] K. Gröchenig: Foundations of time-frequency analysis. Birkhäuser, Boston, 2000.

[9] M. Holschneider: Wavelets. An analysis tool. Oxford Mathematical Monographs. Oxford Science Publications. The Clarendon Press, Oxford University Press, New York, 1995.

[10] A. J. E. M. Janssen: The duality condition for Weyl-Heisenberg frames. In "Gabor analysis: theory and applications" (eds. H.G. Feichtinger and T. Strohmer). Birkhäuser, Boston, 1998.

[11] A. J. E. M. Janssen: Signal analytical proofs of two basic results on lattice expansions. Appl. Comp. Harm. Anal. 1 (1994), 350-354.

[12] R. S. Laugesen: Gabor dual spline windows. Appl. Comput. Harmon. Anal. 27 (2009), 180-194.

[13] S. Li and D. Yan: Frame fundamental sensor modeling and stability of one-sided frame perturbation. Acta Applicandae Mathematicae 107 (2009), 91-103.

[14] A. Ron and Z. Shen: Frames and stable bases for shift-invariant subspaces of $L^{2}\left(\mathbb{R}^{d}\right)$. Canad. J. Math. 47, 1051-1094, 1995.

[15] D. Walnut: Continuity properties of the Gabor frame operator. J. Math. Anal. Appl. 165, 479-504, 1992.

[16] T. Werther, Y. C. Eldar and N. K. Subbanna: Dual Gabor frames: theory and computational aspects. IEEE Trans. Signal Process. 53, 41474158, 2005.

[17] R. Young: An introduction to nonharmonic Fourier series. Academic Press, New York, 1980 (revised first edition 2001). 
Ole Christensen, Department of Mathematics, Technical University of Denmark, Building 303, 2800 Lyngby, Denmark Ole.Christensen@mat.dtu.dk Richard S. Laugesen, Department of Mathematics, University of Illinois at Urbana-Champaign,Urbana, IL 61801,U.S.A. Laugesen@illinois.edu 